\title{
Seguimiento posquirúrgico de los pacientes con poliposis adenomatosa familiar: resultados en una población del sur de España
}

\author{
C. Cordero Fernández, A. Pizarro Moreno, M. Garzón Benavides, R. García Lozanoํㅡ O. Belda Laguna, \\ S. Sobrino, J. M. Bozada y T. Zulueta Dorado²
}

Servicios de Aparato Digestivo, 'Inmunología y ${ }^{2}$ Anatomía Patológica. Hospital Universitario Virgen del Rocío. Sevilla

\section{RESUMEN}

Objetivo: analizar la evolución de la mucosa rectal y del reservorio así como idoneidad de los intervalos de seguimiento y del tratamiento realizado para evitar la aparición del cáncer, en una serie de pacientes con poliposis adenomatosa familiar (PAF), intervenidos.

Método: estudio prospectivo de 28 pacientes con PAF intervenidos mediante anastomosis íleo-rectal (20 pacientes) y anastomosis íleo-anal con reservorio (8 pacientes). A todos se les había realizado un control endoscópico dos veces al año y análisis del número y características macroscópicas e histológicas de los pólipos antes y después de la cirugía así como del tratamiento realizado, de sus complicaciones y de la adecuación del intervalo de seguimiento. El seguimiento medio fue de 6,47 años ( $\mathrm{DE}=4,59$; rango $=0,72-16,75$ años) .

Resultados: ninguno de los 26 pacientes que cumplimentaron correctamente el protocolo de seguimiento desarrolló cáncer. Sólo dos pacientes lo desarrollaron al 1,75 y los 3 años, respectivamente del abandono del protocolo. Los pacientes que desarrollaron adenomas durante el seguimiento fueron tratados con éxito mediante polipectomía endoscópica, salvo en dos casos que se indicó cirugía.

Conclusiones: en nuestra serie, el incumplimiento de las revisiones ha sido el factor que ha condicionado la aparición de cáncer.

Palabras clave: Poliposis adenomatosa familiar. Anastomosis íleo-rectal. Anastomosis íleo-anal con reservorio ileal. Adenomas de colon. Seguimiento posquirúrgico.

\begin{abstract}
Objective: the study was to assess changes in the rectal mucosa and pouch in a series of patients with familial adenomatous polyposis (FAP) who underwent either subtotal colectomy and ileorectal anastomosis (IRA) or proctocolectomy and ileal pouchanal anastomosis (IPAA), and to evaluate the suitability of the follow-up interval and postoperative treatment employed to prevent the development of cancer.

Method: this study involved 28 patients with FAP who underwent IRA $(n=20)$ or IPAA $(n=8)$, and were followed endoscopically over a mean period of 7.47 years. The number and both macroscopic and histological features of polyps before and after surgery, the treatment, and complications were all analyzed. The suitability of the follow-up interval was assessed.

Results: none of the 26 patients who complied with follow-up developed rectal cancer. Two patients developed rectal cancer at 21 and 36 months after withdrawing from the protocol. Except in two cases in which surgery was indicated, patients who developed adenomas during follow-up were treated by endoscopic polypectomy.
\end{abstract}

Conclusions: in our series, the failure to comply with followup examinations was associated with cancer development.

Key words: Familial adenomatous polyposis. Ileorectal anastomosis. Ileal pouch-anal anastomosis. Colonic adenomas. Postoperative follow-up.

Cordero Fernández C, Pizarro Moreno A, Garzón Benavides M, García Lozano R, Belda Laguna O, Sobrino S, Bozada JM, Zulueta Dorado T. Seguimiento de los paciente con poliposis adenomatosa familiar: resultados en una población del sur de España. Rev Esp Enferm Dig 2007; 99: 440-445.

\section{INTRODUCCIÓN}

La poliposis adenomatosa familiar (PAF) es una enfermedad hereditaria de carácter autosómico dominan-

\section{Recibido: 23-10-06.}

Aceptado: 16-03-07.

Correspondencia: Carmen Cordero Fernández. Servicio de Aparato Digestivo. Hospital Universitario Virgen del Rocío. Avda. Manuel Siurot, s/n. 41013 Sevilla. e-mail: carmenc@inicia.es te, causada por una mutación en la línea germinal del gen APC (gen de la poliposis cólica), situado en el cromosoma 5q21 (1). Clásicamente la PAF se ha caracterizado por la presencia de más de 100 pólipos en el colon $\mathrm{y}$ recto, que de forma natural evolucionan indefectiblemente a carcinoma alrededor de la cuarta década de la vida.

Para prevenir el desarrollo del cáncer sería necesario extirpar la mucosa del colon y recto en su totalidad. No existe actualmente un proceder único para lograr ese ob- 
jetivo y, en ningún caso, se cura la enfermedad, que tiene otras manifestaciones neoplásicas extracolónicas.

Los pacientes con PAF son generalmente jóvenes y están asintomáticos por lo que son contrarios a aceptar como tratamiento preventivo la proctocolectomía total con ileostomía definitiva.

Las dos opciones quirúrgicas existentes para ellos son la colectomía subtotal con anastomosis íleo-rectal o IRA (ileo-rectal anastomosis) y la proctocolectomía con anastomosis íleo-anal y reservorio o IPAA (ileal pouch-anal anastomosis). La IRA tiene buenos resultados funcionales y escasas complicaciones. Sin embargo, después de 25 años de seguimiento, el riesgo acumulativo de desarrollar cáncer en el muñón rectal es del 15\%, y alrededor del $40 \%$ de los pacientes así tratados pueden necesitar a lo largo de su vida una proctectomía secundaria o una reconversión a IPAA para tratar pólipos incontrolables (2). La IPAA mantiene la continencia anal, evita la ileostomía, y reduce el riesgo de cáncer rectal. Las desventajas son su mayor complejidad técnica, y el riesgo, aunque menor que el de la IRA, de desarrollar pólipos adenomatosos o cáncer en el propio reservorio, o en los islotes de mucosa rectal remanente (3-5).

En el presente trabajo analizamos la evolución de la mucosa rectal y el reservorio ileal en nuestros pacientes con PAF intervenidos con IRA e IPAA respectivamente, así como la idoneidad del seguimiento y tratamiento que hemos recomendado, y las complicaciones derivadas del mismo.

\section{PACIENTES Y MÉTODOS}

\section{Pacientes}

Entre 1976 y 2004, se diagnosticaron 45 pacientes de 15 familias con PAF en el Servicio de Aparato Digestivo del Hospital Virgen del Rocío de Sevilla. Nuestro hospital tiene una población dependiente de 596.468 personas por lo que el número de pacientes diagnosticados se corresponde con la prevalencia de la PAF en nuestra población. Todos los pacientes fueron incluidos en una base de datos del Servicio.

Treinta y dos de estos pacientes fueron intervenidos quirúrgicamente. El resto de los pacientes están pendientes de intervención pues son aún demasiado jóvenes. De los 32 pacientes, 15 fueron pacientes probandos y 17 familiares diagnosticados mediante test genético y/o colonoscopia. Siete pacientes no tenían historia familiar previa de cáncer o PAF $\mathrm{y}$ por tanto fueron considerados como primeras mutaciones.

\section{Técnica quirúrgica empleada para el tratamiento de la poliposis}

Las intervenciones fueron realizadas por diferentes cirujanos del Servicio de Cirugía Digestiva. Los enfermos intervenidos fueron estudiados previamente y revisados tras la cirugía por un solo miembro de nuestro Servicio dedicado a la prevención del cáncer colorrectal. El tipo de intervención llevada a cabo fue colectomía subtotal y anastomosis íleo-rectal (IRA), proctocolectomía total con anastomosis íleo-anal con reservorio ileal (IPAA) o proctocolectomía con ileostomía definitiva, en función de distintos factores e implicando al gastroenterólogo, al cirujano y al paciente en la decisión. Previamente el paciente fue informado de los riesgos y los beneficios de cada opción.

Un total de 20 enfermos con PAF fueron tratados con IRA, 8 con IPAA y 4 con proctocolectomía total e ileostomía permanente. De estos, en 3 existía ya un cáncer en la unión rectosigmoidea y en el cuarto la indicación la estableció su cirujano.

La IRA era la única opción quirúrgica hasta el año 1993 en que comenzó a realizarse la IPAA en nuestro hospital. A partir de entonces la IPPA se aconsejó a todos aquellos pacientes con más de 20 pólipos en ampolla rectal. Esta decisión se tomo de acuerdo con la opinión de nuestros endoscopistas estableciendo en 20 el número límite a partir del cual podría ser difícil controlar los pólipos rectales con tratamiento endoscópico. Antes de la cirugía, se trataron los pólipos rectales en aquellos enfermos en los que fue posible.

\section{Protocolo de seguimiento}

Tras la intervención quirúrgica, todos los pacientes fueron seguidos de forma prospectiva cumplimentando un protocolo estructurado en el que se recogía: edad al diagnóstico de la poliposis, número de pólipos en ampolla rectal antes de la cirugía, tipo de intervención realizado, y hallazgos de las endoscopias posquirúrgicas, tanto de las rectales como de las orales. Tales hallazgos incluían: número de adenomas, localización, tamaño, tratamiento aplicado (polipectomía, argón plasma o cirugía), histología de los pólipos resecados, y, evolución de los no resecados. El número de pólipos fue agrupado en tres categorías: menos de 10 , entre 10 y 20 y más de 20; el tamaño de los mismos fue agrupado en menos de $5 \mathrm{~mm}$, entre 5 y $10 \mathrm{~mm}$, y mayores de $10 \mathrm{~mm}$. El grado histológico se definió en función del grado de displasia (de bajo o alto grado), y del tipo de carcinoma, intramucoso o invasor. Se consideraron pólipos de riesgo los de tamaño mayor de $10 \mathrm{~mm}$, los vellositarios y aquellos con displasia de alto grado. En cada revisión se solicitó estudio endoscópico cuidadoso de la mucosa rectal remanente, el reservorio y la unión ano-rectal por retrovisión. El estudio endoscópico se realizó con colonoscopios flexibles de fibra o de video según el año de la revisión. El intervalo de seguimiento endoscópico fue de medio año para los enfermos con IRA y de un año para los enfermos con IPAA. 


\section{Tratamiento durante el seguimiento}

Se realizó polipectomía endoscópica en todos los pólipos mayores de $3 \mathrm{~mm}$, y se indicó cirugía en aquellos que no fueron resecables por esta técnica. Los pólipos incipientes se trataron con argón plasma. Cuando se encontraron pólipos de riesgo, se acortó el tiempo de revisión y se realizó terapia endoscópica hasta la total desaparición del tejido neoplásico.

En tres pacientes intervenidos con IRA, se indico proctocolectomía: en 1 caso por la aparición de un pólipo de riesgo en la unión ano-rectal intratable endoscópicamente, y en los otros dos, por el desarrollo de un carcinoma rectal. Uno de estos pacientes había rechazado dos años antes del diagnóstico de carcinoma una reconversión de la IRA en IPAA, recomendada por la existencia de numerosos pólipos en ampolla rectal, difíciles de resecar por endoscopia.

Incidentalmente algún paciente recibió AINE por otra causa.

El seguimiento medio de los pacientes con IRA fue de 7,47 años (rango $=0,72-16,75 ; \mathrm{DE}=4,92$ años) y la de los enfermos con IPAA de 4,05 años (rango $=1,48-7,34$; $\mathrm{DE}=2,55$ años).

\section{RESULTADOS}

La distribución por sexo fue: 19 hombres y 13 mujeres. La edad media del grupo fue de 28,6 años (DE = 10,59; rango $=13-55$ años).

La edad media de los pacientes con IRA fue de 30,15 años $(\mathrm{DE}=10,98$; rango $=13-55$ años $)$. La de los pacientes con IPAA fue de 20,88 años $(\mathrm{DE}=6,60$; rango $=12$ 30 años).

El número de adenomas encontrados en la ampolla rectal antes de la intervención en los 20 pacientes tratados con IRA fue: igual o menor de 10 adenomas en 11 pacientes $(55 \%)$; entre 10 y 20 adenomas en 1 paciente (5\%) y más de 20 adenomas en 8 pacientes (40\%). Estos últimos 8 pacientes fueron intervenidos entre 1976 y 1993 antes de la puesta en marcha de la IPAA en nuestro hospital.

Los 8 pacientes intervenidos con IPAA tenían más de 20 adenomas en la ampolla rectal antes de la intervención, y en 6 de ellos, el 75\%, el número de estos pólipos estaba entre 30 y 100 .

\section{Evolución de los pacientes intervenidos con IRA}

Se practicó IRA a 20 enfermos con PAF. En la primera revisión endoscópica 5 de ellos (25\%) estaban libres de adenomas. Por el contrario en los otros 15 pacientes (75\%) se encontraron adenomas: menos de 10 en 3 (20\%); entre 10 y 20 adenomas en 6 (40\%); y más de 20 adenomas en los 6 restantes (40\%).
Respecto al tamaño de los adenomas encontrados, en 11 de los pacientes (74\%) era menor de $5 \mathrm{~mm}$; en 2 pacientes (14\%) estaba entre 5 y $10 \mathrm{~mm}$; y en los otros 2 (14\%) eran mayores de $10 \mathrm{~mm}$.

En el $100 \%$ de los casos la histología de los pólipos fue de adenomas tubulares, y el grado de displasia leve.

El número, tamaño y grado de displasia de los adenomas encontrados permanecieron constantes o disminuyeron en 14 pacientes (74\%) mientras que en otros 5 (26\%), aumentaron, si bien todos estos pacientes, a excepción de uno, pudieron ser tratados mediante polipectomía endoscópica. En 1 paciente debido al corto periodo de seguimiento estos parámetros no pudieron ser valorados.

El tratamiento de los adenomas encontrados a lo largo de las revisiones fue la polipectomía endoscópica en 12 pacientes $(67 \%)$; polipectomía más coagulación con argón plasma en 4 (22\%); polipectomía más anti-COX2 en $1(6 \%)$ y terapia con anti-COX2 en $1(6 \%)$. Estos dos pacientes recibieron anti-COX2, en un caso por pólipos múltiples duodenales, y por pólipos hiperplásicos en el fundus gástrico en el otro.

Una paciente desarrolló a los 11 años de la intervención un adenoma velloso plano de $10 \mathrm{~mm}$ de diámetro, con displasia de alto grado, en la unión ano-rectal. Debido a la recurrencia de la lesión tras dos intentos de polipectomía endoscópica, se indicó para su tratamiento proctectomía con ileostomía definitiva, pero la existencia de una intensa fibrosis pélvica muy vascularizada impidió la resección de la ampolla rectal, practicándose finalmente ileostomía definitiva y cierre de muñón rectal. La lesión polipoide recidivó en dos ocasiones más tras sendas polipectomías endoscópica transanales, si bien, con un grado menor de displasia. Finalmente la enferma desarrolló una estenosis de la ampolla rectal que impidió el seguimiento vía endoscópica. A los 7 años de dicha estenosis de la ampolla rectal, la paciente se encontraba asintomática, y sin evidencia de lesión en los controles de seguimiento realizados por estudios de imagen: tomografía axial computarizada (TAC) y resonancia magnética (RM) pélvicas.

Otra paciente con poliposis adenomatosa familiar atenuada (PAFA), diagnosticada sobre la base de la existencia de hipertrofia congénita del epitelio pigmentario de la retina, presencia de más de 100 pólipos hiperplásicos en fundus gástrico, y de unos 20 pólipos mínimos situados en colon derecho, sin pólipos rectales, desarrolló, antes de indicar la intervención, un adenoma tubular de 10 × 6 $\mathrm{mm}$ con displasia de bajo grado en la unión ano-rectal. Este pólipo diagnosticado al hacer retrovisión se pudo extirpar por completo. Las revisiones posteriores fueron normales. Tras 8 años de seguimiento se realizó una IRA. En el momento de la intervención había a lo largo de todo el colon unos 50 pólipos todos ellos menores de $10 \mathrm{~mm}$. No había ningún pólipo en la ampolla rectal y en los 3 años de seguimiento posquirúrgico no se han encontrado pólipos a ese nivel. 
En 2 pacientes (el 11\%) se diagnosticó un carcinoma de recto a los 16 y 17 años de la intervención. Ambos, por problemas familiares y personales, habían abandonado el seguimiento 1,75 y 3 años antes del diagnóstico de cáncer y por lo tanto habían dejado de hacer 3 y 6 revisiones respectivamente. Uno de ellos tenía en ampolla rectal desde la intervención, a pesar de las polipectomías repetidas, más de 30 pólipos menores de $5 \mathrm{~mm}$, razón por la que se le había propuesto reconversión de IRA en IPAA, que rechazó 1 año antes de abandonar el seguimiento. En la primera exploración realizada tras el abandono del seguimiento se encontró un adenocarcinoma sobre un pólipo de $2,5 \mathrm{~cm}$ en estadio T2N0M0. Se trató mediante resección abdomino-perineal. Falleció varios años después por otra causa. El otro paciente desarrolló adenocarcinoma sobre un pólipo de $3 \mathrm{~cm}$ en estadio T3NOM0. Tenía además unos 25 pólipos menores de $5 \mathrm{~mm}$ en ampolla rectal. Fue tratado mediante radioterapia y resección abdómino-perineal, falleciendo a los 24 meses de la intervención con una recidiva local.

\section{Evolución de los pacientes con PAF tratados con IPAA}

Se realizó IPAA en 8 pacientes. En ninguno de ellos se realizó mucosectomía total. En todos quedó un rodete de mucosa rectal remanente de unos $3 \mathrm{~cm}$ de longitud media (valores extremos de 1 y $5 \mathrm{~cm}$ ).

En la primera endoscopia control realizada tras la intervención se encontraron adenomas en el remanente de mucosa rectal en 2 de los 8 pacientes (25\%). En ambos casos el número de adenomas fue menor de 10; todos ellos fueron adenomas tubulares y de tamaño inferior a $5 \mathrm{~mm}$ de diámetro.

En la segunda endoscopia control aparecieron adenomas en otros dos pacientes, siendo similares en número, tipo histológico y tamaño a los antes mencionados.

En las siguientes revisiones en un paciente (14\%) disminuyó el número de adenomas y en otros tres (43\%) no hubo cambios en cuanto al número y características de los mismos.

El tratamiento de los adenomas fue en todos los casos la polipectomía endoscópica. En ningún caso se desarrolló adenocarcinoma, ni pólipos de riesgo.

Dos pacientes de nuestra serie sufrieron complicaciones derivadas de la polipectomía: Uno de ellos, tenía una enfermedad de Von Willebrand y tuvo en varias ocasiones hemorragias leves tras la polipectomía que se resolvieron de forma espontánea. Un segundo paciente, tras polipectomías repetidas y legrado de la mucosa rectal, desarrolló una estenosis intratable de ampolla rectal.

\section{DISCUSIÓN}

En la actualidad el tratamiento aceptado para los pacientes con PAF es la intervención quirúrgica mediante
IRA o IPAA, con un seguimiento endoscópico posterior, imprescindible para prevenir el desarrollo de cáncer que con distinta frecuencia puede aparecer en la mucosa rectal remanente o en el reservorio, según el tipo de intervención realizada.

El riesgo estimado de desarrollar cáncer en la mucosa rectal, varía mucho según el año de publicación de las distintas series. Así en 1971 fue del 59\% a los 23 años de seguimiento (6), 10 años más tarde del $32 \%$ a los 20 años (7) y en series más recientes se cifra en el $13 \%$ a los 25 años (8), y en el $12 \%$ a los 20 años de seguimiento (9). Desde la puesta en marcha de la IPAA, parece probable que esta frecuencia disminuya notablemente ya que la indicación de IRA se hace de forma selectiva, tal como demuestran la serie de la Cleveland Clinic publicada recientemente (29).

En nuestra serie, el $40 \%$ de los pacientes tratados con IRA tenían, antes de la intervención más de 20 adenomas en ampolla rectal y de ellos, el $75 \%$ presentaron pólipos en la primera revisión endoscópica realizada a medio año después de la intervención. Si bien en su mayoría estos pólipos eran pequeños, en dos casos alcanzaron tamaños mayores de $10 \mathrm{~mm}$, lo que no impidió su tratamiento con polipectomía endoscópica. A pesar de ello, ninguno de los pacientes que cumplimentaron el protocolo de seguimiento y tratamiento propuesto, llegó a desarrollar cáncer a lo largo de todo el tiempo de seguimiento.

Sólo dos pacientes de nuestra serie, el 11,7\%, desarrollaron cáncer en la ampolla rectal. Ambos habían abandonado el seguimiento endoscópico y el diagnóstico se hizo en la primera endoscopia realizada a 1,75 y 3 años respectivamente después de interrumpir el seguimiento. Si bien ambos enfermos tenían un número muy elevado de pólipos rectales antes de la cirugía y un tiempo transcurridos desde la intervención de más de 15 años, el hecho de que sean los dos únicos casos de cáncer de nuestra serie y los únicos de los 20 pacientes que incumplieron el protocolo de seguimiento, nos lleva a pensar que fue el incumplimiento de las revisiones el factor que condicionó la aparición del cáncer. Ninguno de los otros pacientes de nuestra serie con un elevado número de pólipos rectales antes de la cirugía desarrollaron cáncer, como tampoco lo han desarrollado otros pacientes que tenían algunos de los otros factores de riesgo mencionados en la literatura $(6,10-13)$.

Es interesante señalar el hallazgo en dos pacientes de pólipos planos en la unión ano-rectal, uno de ellos con displasia de alto gado, que pasaron desapercibidos por visión frontal, y cuyo tratamiento precoz, con toda probabilidad evitó el desarrollo de un carcinoma.

Los resultados de nuestra serie parecen indicar la eficacia de nuestro protocolo de seguimiento, con intervalos de 6 meses, exploración meticulosa de la unión ano-rectal por retrovisión y tratamiento endoscópico de las lesiones. La posibilidad de que nuestros resultados estén en parte condicionados por el relativo corto tiempo de seguimiento, no puede ser rotundamente descartada, pero creemos 
significativo que el incumplimiento del protocolo haya sido en nuestra serie el único factor asociado al desarrollo de cáncer rectal.

Dos pacientes de la serie recibieron tratamiento con AINE. Uno, para el control de pólipos duodenales y el otro, un paciente con PAFA, para intentar controlar los pólipos del fundus gástrico (14). El empleo de Sulindac como terapia de los pólipos rectales ha quedado en entredicho con la publicación de 4 casos de cáncer rectal en pacientes con IRA, previamente tratados con Sulindac, y con aparente desaparición de los pólipos en revisiones previas (15-20). Este dato y la modificación que sufren los pólipos tras el mencionado tratamiento, que dificulta su posterior reconocimiento y tratamiento, nos ha llevado a no utilizar AINE en el control de los pólipos rectales de nuestros pacientes.

La IPAA, una intervención aún reciente, está universalmente aceptada ya que el riesgo de cáncer de recto es significativamente más bajo que en la IRA. No obstante, tiene una tasa global de complicaciones del $24 \%$, entre las que se describen la obstrucción de intestino delgado, la sepsis pélvica, la disfunción sexual masculina y femenina, el fallo del reservorio y la reservoritis (21-29) y no es desdeñable el riesgo de desarrollar pólipos neoplásicos y cáncer en el reservorio o en islotes de mucosa rectal retenidos, de los que hay ya numerosos casos descritos (9,30-38).

La gravedad de estas lesiones neoplásicas parece estar en relación con el tiempo transcurrido desde la intervención, con un intervalo medio entre la cirugía de la poliposis y la aparición de adenomas en el reservorio de 4,7 años (0,15-12 años) (31). La importancia de este tipo de lesiones está aún por determinar.

En nuestra serie, en la que hemos seguido a los pacientes con PAAF una media de 4,05 años, no hemos encontrado ningún caso de cáncer en la anastomosis íleo-anal ni en la mucosa rectal remanente. El 50\% de los pacientes han desarrollado pólipos en el rodete de mucosa rectal dejado tras la intervención, pero estas lesiones siempre fueron de bajo riesgo y pudieron ser tratadas endoscópicamente. No hemos encontrado pólipos en el reservorio al cierre de este estudio, aunque recientemente hemos comenzado a realizar tinción del reservorio con colorantes vitales y en dos pacientes se han puesto de manifiesto múltiples pólipos de pequeño tamaño no visibles antes de la tinción.

La eficacia de las revisiones mejorará probablemente con el uso de endoscopios de magnificación y de tinciones vitales, que permitan identificar pequeñas lesiones neoplásicas así como cada una de las zonas del canal anal y las zonas de metaplasia intestinal no visibles con los endoscopios convencionales.

\section{CONCLUSIONES}

El objetivo más importante en la elección del tratamiento quirúrgico de los enfermos con PAF es la preven- ción del cáncer colorrectal. La elección del tipo de intervención debe tener en cuenta los factores de riesgo de cada paciente, su comprensión de la enfermedad, y sus características personales.

La IRA sigue siendo una buena opción terapéutica para los pacientes jóvenes de bajo riesgo, siempre que puedan seguir las revisiones periódicas aconsejadas.

Las revisiones periódicas incluyen:

- La exploración minuciosa cada 6 meses del muñón rectal en los pacientes con IRA y del reservorio y anastomosis íleo-anal en los intervenidos mediante la IPAA.

-El estudio sistemático por retrovisión de la unión ano-rectal.

En nuestra experiencia ninguno de los pacientes que cumplimentaron las revisiones establecidas desarrollaron cáncer de la mucosa rectal ni reservorio. De ahí la importancia de que el paciente comprenda la necesidad ineludible de las revisiones periódicas.

La polipectomía ha sido el tratamiento más utilizado durante las revisiones periódicas y en la mayoría de los pacientes ha logrado la disminución en el número, tamaño y grado histológico de los pólipos.

Sólo ha habido dos complicaciones derivadas de la terapéutica: una hemorragia muy leve, aunque repetida pospolipectomía que cedió de forma espontánea sin necesitar tratamiento y una estenosis de ampolla intratable cuyas consecuencias habrá que determinar en años venideros.

\section{BIBLIOGRAFÍA}

1. Nyam DC, Brillant PT, Dozois RR, Kelly KA, Pemberton JH, Wolff BG. Ileal pouch-anal canal anastomosis for familial adenomatous polyposis. Early and late results. Ann Surg 1997; 226 (4): 514-21.

2. Rodríguez Sanjuán JC, Casanova Rituerto D, Martino Fernández E, Fernández Escalante JC, Gómez Fleitas JM, Herrera Noreña L, et al. Poliposis adenomatosa familiar: Proctocolectomía con reservorio ileal frente a preservación rectal. Rev Esp Enferm Dig 1992; 82 (3): $159-63$.

3. Herbay A, Stern J, Herfarth C. Pouch-anal cancer after restorative proctocolectomy for familial adenomatous polyposis. Am J Surg Pathol 1996; 20 (8): 995-9.

4. Duijvendijk P, Slors FM, Taat CW, Oosterveld P, Vasen H. Functional outcome after colectomy and ileorectal anastomosis compared with proctocolectomy and ileal pouch anal anastomosis in familial adenomatous polyposis. Ann Surg 1999; 230 (5): 648-54.

5. Ambroze WL, Dozoiis RR, Pemberton JH, Beart RW, Ilstrup DM. Familial adenomatous polyposis: Results following ileal pouch-anal anastomosis and ileorectostomy. Dis Colon Rectum 1992; 35 (1): 12-5.

6. Moertel CG, Hill JR, Adson MA. Management of multiple polyposis of the large bowel. Cancer 1971; 28: 160-1.

7. Bess RW Jr. Familial polyposis. Br J Surg 1985; 72: 831-2.

8. Bussey HJR, Eyers AA, Ritchie SM, Thomson JPS. The rectum in adenomatous polyposis: The St. Mark's policy. Br J Surg 1985; 72: 829-31.

9. Sarre RG, Jagelman DJ, Beck GJ, McGannon E, Fazio VW, Weakley FL, et al. Colectomy with ileo-rectal anastomosis for familial adenomatous polyposis: the risk of rectal cancer. Surgery 1987; 101: 20-6.

10. Harvey JC, Quan SHQ, Stearns MW. Management of familial polyposis with preservation of the rectum. Surgery 1978; 84: 476-82.

11. Nikitin AM, Obukhov VK, Chubarov YY, Jakushin AV. Results of a thirty years study of familial adenomatous polyposis coli. Dis Colon Rectum 1997; 40 (6): 679-84.

12. Matsuo S, Eguchi S, Azuma T, Hidaka M, Yamaguchi S, Hayashi T, et al. Attenuated familial adenomatous polyposis associated with ad- 
vanced rectal cancer in a 16 year old boy: Report of a case. Surg Today 2001; 31 (11): 1020-3.

13. Vasen HF, van der luijt RB, Slors JF, Buskenns E, de Ruiper P, Baeten CG. Molecular genetic testing as guide to surgical management of familial adenomatous polyposis. Lancet 1996; 348: 433-5.

14. Zigau J, Piqué JM, Rubio E, Planas RT, Bordas JM. Effects of longterm Sulindac therapy on colonic polyposis. Ann Intern Med 1991; 115: 952-4.

15. Vasen HFA, Duijvendijk P, Buskens E, Bulow C, Bjork J, Jarvinen HJ, et al. Decision analysis in teh surgical treatmento of patients with familial adenomatous polyposis: A dutch Scandinavian collaborative study including 659 patients. Gut 2001; 49: 231-5.

16. Niv Y, Fraser GM. Adenocarcinoma in the rectal segment in familial polyposis coli is not pervented by sulindac therapy. Gastroenterology 1994; 107: 854-57.

17. Lynch HT, Thorson AG, Smyrk T. Rectal cancer after prologed sulindac chemoprevention. Cancer 1995; 75 (4): 936-8.

18. Tonelli F, Valanzano R, Messerini L, Ficari F. Long term treatment with sulindac in familial adenomatous polyposis: Is there an actual efficacy in prevention of rectal cancer? J Surg Oncol 2000; 74 (1): 15-20.

19. Thorson AG, Lynch HT, Smyrk TC. Rectal cancer in FAP patient after sulindac. Lancet 1994; 343 (8894): 180.

20. Lynch HT, Thorson AG, Smyrk T. Rectal cancer after prolonged sulindac chemoprevention. A case report. Cancer 1995; 75 (4): 936-8.

21. Utech M, Bruwer M, Buerger H, Tubergen D, Senninger N. Rectal carcinoma in a patient with adenomatous polyposis coli after colectomy with ileorectal anastomosis and consecutive chemoprevention with sulindac suppositories. Chirug 2002; 73 (8): 855-8.

22. Gorgun E, Remzi FH, Goldberg JM, Thornton J, Bast J, Hull TL, et al. Fertility is reduced after restorative proctocolectomy with ileal pouch anal anastomosis: A study of 300 patients. Surgery 2004; 136 (4): 795-803.

23. Van Laarhoven CJ, Hueting WE, Schipper ME, Oostvogel HJ, Akkermams LM, van Vroonhoven TJ, et al. Ileo neorectal anastomosis: Medium and long-term follow up of 37 patients. Dig Surg 2004; 20: 21 (5): 371-9.

24. Lepisto A, Luukkonen P, Jarvinen HJ. Cumulative failure rate of ileal pouch anal anastomosis and quality of live after failure. Dis Colon Rectum 2002; 45 (10): 1289-94.

25. Delaney CP, Fazio VW, Remzi FH, Hammel J, Church JM, Hull TL, et al. Prospective, age-related analysis of surgical results funtional outcome, and quality of life after ileal pouch-anal anastomosis. Ann Surg 2003; 238 (2): 221-8.

26. Fazio VW, Tekkis PP, Remzi FH, Lavery IC, Manililch E, Connor J, et al. Quantification of risk for pouch failure after ileal pouch anal anastomosis surgery. Ann Surg 2003; 238 (4): 605-17.

27. Duijvendijk P, Slors FM, Taat CW, Oosterveld P, Vasen H. Functional outcome after colectomy and ileorectal anastomosis compared with proctocolectomy and ileal pouch anal anastomosis in familial adenomatous polyposis. Ann Surg 1999; 230 (5): 648-54.

28. Ambroze WL, Dozoiis RR, Pemberton JH, Beart RW, Ilstrup DM. Familial adenomatous polyposis: Results following ileal pouch-anal anastomosis and ileorectostomy. Dis Colon Rectum 1992; 35 (1): 12-5.

29. Church J, Burke C, McGannon E, Pastean O, Clark B. Risk of rectal cancer in patients after colectomy and ileorectal anastomosis for familial adenomatous polyposis: a funtion of available surgical options. Dis Colon Rectum 2003; 46 (9): 1175-81.

30. Church J. Ileoanal pouch neoplasia in familial adenomatous polyposis: An underestimated threat. Dis Colon Rectum 2005; 48 (9): 170813.

31. Campos FG, Habr-Gama A, Kiss DR, Vieira de Silva E, Rawet V, Imperiale AR, et al. Adenocarcinoma after ileoanal anastomosis for familial adenomatous polyposis: Review of risk factors and currente surveillance apropos of a case. J Gastrointest Surg 2005; 9 (5): 695702 .

32. Vuilleumier H, Halkic N, Ksontini R, Gillet M. Columnar cuff cancer alter restorative proctocolectomy for familial adenomatous polyposis. Gut 2000; 47: 732-4.

33. Wu JS, Mc Gannon ES, Curch JM. Incidente of neoplastic polyps in the ileal pouch of patients with familial adenomatous polyposis after restorative proctocolectomy. Dis Colon Rectum 1998; 41: 552-7.

34. Thompson Fawcett MW, Marcus VA, Redston M, Cohen Z, McLeod RS. Adenomatous polyps develop comonly in the ileal pouch of patients with familial adenomatous polyposis. Dis Colon Rectum 2001; 44: 347-53.

35. Parc YR, Olschwang S, Dsaint B, Schmitt G, Parc RG, Tiret E. Familial adenomatous polyposis: Prevalence of adenomas in the ileal pouch after restorative proctocolectomy. Ann Surg 2001; 233: 3604.

36. Bassuini MM, Billings PJ. Carcinoma in an ileoanal pouch after restorative proctocolectomy for familial adenomatous polyposis. $\mathrm{Br} \mathrm{J}$ Surg 1996; 83: 506.

37. Palkar VM, deSouza LJ, Jagannath P, Naeresh KN. Adenocarcinoma arising in $\mathrm{J}$ pouch after total proctocolectomy for familial polyposis coli. Indian J Cancer 1997; 34: 16-9.

38. Curch J, Burke C, McGannon E, Pastean O, Clark B. Predicting polyposis severity by proctoscopy. How reliable is it? Dis Colon Rectum 2001; 44 (9): 1249-52. 